\title{
Asymptotic stability of homogeneous states in the relativistic dynamics of viscous, heat-conductive fluids
}

\author{
Matthias Sroczinski
}

July 4, 2017

\begin{abstract}
This paper shows global-in-time existence and asymptotic decay of small solutions to the Navier-Stokes-Fourier equations for a class of viscous, heat-conductive fluids. As this second-order system is symmetric-hyperbolic, existence and uniqueness on a short time interval follow from work of Hughes, Kato, and Marsden. Here it is proven that solutions which are close to a homogeneous reference state can be extended globally and decay to the reference state. The proof combines decay results for the linearization with refined Kawashima-type estimates of the nonlinear terms.
\end{abstract}

\section{Introduction}

In relativistic fluid dynamics, stresses in perfect fluids are described by the inviscid energy-momentum-tensor ("relativistic inviscid Cauchy Tensor")

$$
T^{\alpha \beta}=(\rho+p) u^{\alpha} u^{\beta}+p g^{\alpha \beta},
$$

where $\rho$ and $p$ are the internal energy and the pressure of the fluid and $u^{\alpha}$ is its 4 -velocity. In this paper we will exclusively consider causal barotropic fluids, a class defined by the property that there exists a one-to-one relation between $\rho$ and $p$,

$$
p=\hat{p}(\rho),
$$


with a smooth function $\hat{p}:(0, \infty) \rightarrow(0, \infty)$ that satisfies $0<\hat{p}^{\prime}<1$. One way to describe the dynamics of dissipative barotropic fluids is via a system

$$
\frac{\partial}{\partial x^{\beta}}\left(T^{\alpha \beta}+\Delta T^{\alpha \beta}\right)=0, \alpha=0,1,2,3
$$

of partial differential equations - the conservation laws of energy and momentum -, in which the "dissipation tensor" $\Delta T^{\alpha \beta}$ is linear in the gradients of the state variables determined by coefficients $\eta, \zeta$ of viscosity and $\chi$ of heat conduction. Freistühler and Temple have recently proposed a particular new way of choosing $\Delta T^{\alpha \beta}$ such that basic requirements, notably of causuality, are met; see [3] for this and also for a discussion of the interesting history of the causuality problem. According to [3], $\Delta T^{\alpha \beta}$ is given as

$$
-\Delta T^{\alpha \beta}=B^{\alpha \beta \gamma \delta}(\psi) \frac{\partial \psi_{\gamma}}{\partial x^{\delta}},
$$

where $\psi$ denotes the so-called Godunov variables

$$
\psi_{\gamma}=\frac{u_{\gamma}}{f}
$$

with $f$ the Lichnerowicz index of the fluid. The key property of Godunov variables is that in these, the first-order term of a system of conservation laws, here

$$
\frac{\partial}{\partial x^{\beta}} T^{\alpha \beta},
$$

becomes symmetric hyperbolic [4]. ${ }^{1}$ Now, the requirement that also

$$
-\frac{\partial}{\partial x^{\beta}}\left(\Delta T^{\alpha \beta}\right)
$$

should be symmetric hyberbolic when written in the same variables determines a set of coefficient fields $B^{\alpha \beta \gamma \delta}(\psi)$ which make (1.3) an element of a class of systems that was introduced by Hughes, Kato and Marsden and shown to be well-posed in Sobolev spaces [5]. As shown in [3], the requirements of equivariance (isotropicity) and other physical necessities indeed make $B^{\alpha \beta \gamma \delta}(\psi)$ determined by the coefficients $\eta, \zeta, \chi$.

The purpose of this paper is to provide a global-in-time solution theory of these relativistic Navier-Stokes-Fourier equations (1.3). To this end, we analyze first the linearization of (1.3) at some homogeneous reference

\footnotetext{
${ }^{1}$ See [2] for details and the history of the use of such variables in relativistic fluid dynamics
} 
state and then the nonlinear problem as a pertubation of the linear one, both with techniques that were developed, or are simililar to techniques developed by Kawashima and co-authors notably in [6], [1].

To have a clear setting, we carry out the whole argument under the additional assumption that the fluid is indeed thermobarotropic, which means that that not only its pressure but also its temperature is a function of its internal energy,

$$
\theta=\hat{\theta}(\rho)
$$

In this case, the Lichnerowicz index is identical with the temperature,

$$
f=\theta \text {. }
$$

and actual heat conduction can be an integrated part of a four-field theory, see [2]. An important physical example of this is given by the case of the pure radiation fluid [7], whose internal energy as function of particle number, density and specific entropy is given by

$$
\rho(n, s)=k n^{\frac{4}{3}} s^{\frac{4}{3}} .
$$

\section{Preliminaries and Notation}

As stated in the introduction, the goal of this paper is to prove existence and asymptotic decay of global-in-time solutions of (1.3) near homogeneous reference states. First, writting (1.3) in Godunov variables gives

$$
\begin{array}{r}
-B^{\alpha \beta \gamma \delta}(\psi) \frac{\partial \psi_{\gamma}}{\partial x^{\beta} \partial_{x}^{\delta}}+\frac{\partial}{\partial x^{\beta}} T^{\alpha \beta}(\psi)-\frac{\partial}{\partial x^{\beta}}\left(B^{\alpha \beta \gamma \delta}(\psi)\right) \frac{\partial \psi_{\gamma}}{\partial x^{\delta}}=0 \\
\alpha=0,1,2,3 .
\end{array}
$$

In our case of a thermobarotropic fluid the "dissipation tensor" and the inviscid energy-momentum-tensor are given by ${ }^{2}$

$$
\begin{aligned}
B^{\alpha \beta \gamma \delta}=\chi \theta^{2} u^{\alpha} u^{\gamma} g^{\beta \delta}-\sigma \theta u^{\beta} u^{\delta} \Pi^{\alpha \gamma}+\tilde{\zeta} \theta \Pi^{\alpha \beta} \Pi^{\gamma \delta} & \\
& +\eta \theta\left(\Pi^{\alpha \gamma} \Pi^{\beta \delta}+\Pi^{\alpha \delta} \Pi^{\beta \gamma}-\frac{2}{3} \Pi^{\alpha \beta} \Pi^{\gamma \delta}\right) \\
& +\sigma \theta\left(u^{\alpha} u^{\beta} \eta^{\gamma \delta}-u^{\alpha} u^{\delta} \eta^{\beta \gamma}\right)+\chi \theta^{2}\left(u^{\beta} u^{\gamma} \eta^{\alpha \delta}-u^{\gamma} u^{\delta} \eta^{\alpha \beta}\right)
\end{aligned}
$$

\footnotetext{
${ }^{2}$ We use the Minkowski metric $g^{\alpha \beta}$ with signature -+++ and the standard projection $\Pi^{\alpha \beta}=g^{\alpha \beta}+u^{\alpha} u^{\beta}$
} 
with $\sigma=\left(\frac{4}{3} \eta+\zeta\right) /\left(1-c_{s}^{2}\right)-c_{s}^{2} \chi \theta, \tilde{\zeta}=\zeta+c_{s}^{2} \sigma-c_{s}^{2}\left(1-c_{s}^{2}\right) \chi \theta$, where $c_{s}^{2}=p^{\prime}(\rho) \in(0,1)$ is the speed of sound (cf. [3]), and

$$
\frac{\partial}{\partial x^{\beta}} T^{\alpha \beta}=\operatorname{sn} \theta^{2}\left[u^{\alpha} g^{\beta \gamma}+u^{\beta} g^{\alpha \gamma}+u^{\gamma} g^{\alpha \beta}+\left(3+c_{s}^{-2}\right) u^{\alpha} u^{\beta} u^{\gamma}\right] \frac{\partial \psi_{\gamma}}{\partial x^{\beta}},
$$

with particle number $n$ and specific entropy $s$. It was shown in [3] that (2.1) is symmetric hyperbolic in the sense of Hughes-Kato-Marsden [5]. Thus, when replacing

$$
B^{\alpha \beta \gamma \delta}(\psi) \frac{\partial \psi_{\gamma}}{\partial x^{\beta} \partial_{x}^{\delta}}
$$

by

$$
\tilde{B}^{\alpha \beta \gamma \delta}(\psi) \frac{\partial \psi_{\gamma}}{\partial x^{\beta} \partial_{x}^{\delta}}=\frac{1}{2}\left(B^{\alpha \beta \gamma \delta}(\psi)+B^{\alpha \delta \gamma \beta}(\psi)\right) \frac{\partial \psi_{\gamma}}{\partial x^{\beta} \partial_{x}^{\delta}},
$$

we can write $(2.1)$ as

$$
A(\psi) \psi_{t t}-\sum_{i, j=1}^{3} B_{i j}(\psi) \psi_{x_{i} x_{j}}+\sum_{j=1}^{3} D_{j}(\psi) \psi_{t x_{j}}+f\left(\psi, \psi_{t}, \partial_{x} \psi\right)=0
$$

where

$$
\begin{aligned}
A & =\left(-\tilde{B}^{\alpha 0 \gamma 0}\right)_{0 \leq \alpha, \gamma \leq 3}, \quad B_{i j}=\left(-\tilde{B}^{\alpha i \gamma j}\right)_{0 \leq \alpha, \gamma \leq 3}, \\
D_{j} & =\left(-\tilde{B}^{\alpha 0 \gamma j}\right)_{0 \leq \alpha, \gamma \leq 3},
\end{aligned}
$$

are symmetric $4 \times 4$ matrices, $A(\psi)$ is positive definite, $\sum_{i, j=1}^{3} \xi_{i} B_{i j}(\psi) \xi_{j}$ is positive definite for arbitrary $\xi \in \mathbb{R}^{3} \backslash\{0\}$, and

$$
f^{\alpha}=\frac{\partial}{\partial x^{\beta}} T^{\alpha \beta}(\psi)-\frac{\partial}{\partial x^{\beta}}\left(B^{\alpha \beta \gamma \delta}(\psi)\right) \frac{\partial \psi_{\gamma}}{\partial x^{\delta}}=0, \alpha=0,1,2,3 .
$$

Throughout the paper we will consider the Cauchy problem associated with (2.2):

$$
\begin{aligned}
A \psi_{t t}-\sum_{i, j=1}^{3} B_{i j} \psi_{x_{i} x_{j}}+\sum_{j=1}^{3} D_{j} \psi_{t x_{j}}+f & =0 \text { on }(0, T] \times \mathbb{R}^{3}, \\
\psi(0) & ={ }^{0} \psi \text { on } \mathbb{R}^{3}, \\
\psi_{t}(0) & ={ }^{1} \psi \text { on } \mathbb{R}^{3},
\end{aligned}
$$

The main result is the following: 
2.1 Theorem. Let $s \geq 4, \theta>0$ and $\bar{\psi}=\left(\theta^{-1}, 0,0,0,\right)^{t}$. Then there exist $\delta_{0}>0, C_{0}=C_{0}(\delta)>0$ such that for all initial data $\left({ }^{0} \psi,{ }^{1} \psi_{1}\right) \in$ $\left(H^{s+1} \times H^{s}\right) \cap\left(L^{1} \times L^{1}\right)$ satisfying $\left\|\left({ }^{0} \psi-\bar{\psi},{ }^{1} \psi\right)\right\|_{s+1, s, 1}^{2}<\delta_{0}$ there exists a unique solution $\psi$ of the Cauchy problem (2.3)-(2.5) such that

$$
\psi-\bar{\psi} \in \bigcap_{j=1}^{s} C^{j}\left([0, \infty), H^{s+1-j}\right) .
$$

$\psi$ satisfies the decay estimates

$$
\begin{gathered}
\|\left(\psi(t)-\bar{\psi}, \psi_{t}(t)\left\|_{s+1, s}^{2}+\int_{0}^{t}\right\|\left(\psi(\tau), \psi_{t}(\tau)\right)\left\|_{s+1, s}^{2} d \tau \leq C_{0}\right\|\left({ }^{0} \psi-\bar{\psi},{ }^{1} \psi\right) \|_{s+1, s}^{2},\right. \\
\left\|\left(\psi(t)-\bar{\psi}, \psi_{t}(t)\right)\right\|_{s, s-1} \leq C_{0}(1+t)^{-\frac{3}{4}}\left\|\left({ }^{0} \psi-\bar{\psi},{ }^{1} \psi\right)\right\|_{s, s-1,1}
\end{gathered}
$$

for all $t \in[0, \infty)$.

In the last part of this section we introduce some notation. For $p \in[1, \infty]$ and some $m \in \mathbb{N}$ just write $L^{p}$ for $L^{p}\left(\mathbb{R}^{3}, \mathbb{R}^{m}\right)$. For $s \in \mathbb{N}_{0}$ we denote by $H^{s}$ the $L^{2}$-Sobolev-space of order $s$, namely

$$
H^{s}:=\left\{u \in L^{2}: \forall \alpha \in \mathbb{N}_{0}^{n}(|\alpha| \leq s):\left\|\partial_{x}^{\alpha} u\right\|_{L^{2}}<\infty\right\}
$$

with norm

$$
\|u\|_{s}=\left(\sum_{0 \leq|\alpha| \leq s}\left\|\partial_{x}^{\alpha} u\right\|_{L^{2}}\right)^{\frac{1}{2}} .
$$

We just write $\|u\|$ instead of $\|u\|_{0}$. For $s, k \in \mathbb{N}_{0}$ and $U=\left(u_{1}, u_{2}\right) \in H^{s} \times H^{k}$ set

$$
\|U\|_{s, k}=\left(\left\|u_{1}\right\|_{s}^{2}+\left\|u_{2}\right\|_{k}^{2}\right)^{\frac{1}{2}}
$$

and for $U \in\left(H^{s} \times H^{k}\right) \cap\left(L^{p}\right)^{2}$ set

$$
\|U\|_{s, k, p}=\|U\|_{s, k}+\|U\|_{\left(L^{p}\right)^{2}} .
$$

For $u \in H^{s}$ and integers $0 \leq k \leq s, \partial_{x}^{k}$ shall denote the vector in $\mathbb{R}^{N}$, $N=m \#\left\{\alpha \in \mathbb{N}_{0}^{n}:|\alpha|=k\right\}$, whose entries are the partial derivatives of $u$ of order $k$.

For $u \in H^{s}, v \in H^{l-1}(0 \leq l \leq s)$ and $\alpha \in \mathbb{N}_{0}^{n},|\alpha| \leq s$ set

$$
\left[\partial_{x}^{\alpha}, u\right] v=\partial_{x}^{\alpha}(u v)-u \partial_{x}^{\alpha} v .
$$

For $\delta>0$ let $\phi_{\delta}$ denote the Friedrichs mollifier and set

$$
\left[\phi_{\delta} *, u\right] v=\phi_{\delta} *(u v)-u\left(\phi_{\delta} * v\right) .
$$




\section{Decay Estimates for the Linearized System}

In this section we study the linearization of (2.2) in the fluids restframe $u^{\alpha}=(1,0,0,0)^{t}$ and for a constant temperature $\theta>0$. The resulting equations read

$$
A^{(1)} \psi_{t t}-\sum_{i, j=1}^{3} B_{i j}^{(1)} \psi_{x_{i} x_{j}}+a^{(1)} \psi_{t}+\sum_{j=1}^{3} b_{j}^{(1)} \psi_{x_{j}}=0,
$$

where

$$
\begin{gathered}
A^{(1)}=\left(\begin{array}{cc}
\chi \theta^{2} & 0 \\
0 & \sigma \theta I_{3}
\end{array}\right), \\
B_{i j}^{(1)}=\left(\begin{array}{cc}
\chi \theta^{2} & 0 \\
0 & \theta \eta I_{3} \delta_{i j}+\theta\left(\tilde{\zeta}+\frac{1}{3} \eta\right)\left(e_{i} \otimes e_{j}+e_{j} \otimes e_{i}\right)
\end{array}\right), \\
a^{(1)}=n s \theta^{2}\left(\begin{array}{cc}
c_{s}^{-2} & 0 \\
0 & I_{3}
\end{array}\right), \quad b_{j}^{(1)}=n s \theta^{2}\left(e_{j} \otimes e_{0}+e_{0} \otimes e_{j}\right),
\end{gathered}
$$

First, multiply (3.1) by $(n s)^{-1} \theta^{-2}$ and set $\bar{\chi}=\chi(n s)^{-1}, \bar{\eta}=\eta(n s \theta)^{-1}$, $\bar{\zeta}=\tilde{\zeta}(n s \theta)^{-1}, \bar{\sigma}=\bar{\zeta}+\frac{1}{3} \bar{\eta}$. We arrive at the equivalent system

$$
A^{(2)} \psi_{t t}-\sum_{i, j=1}^{3} B_{i j}^{(2)} \psi_{x_{i} x_{j}}+a^{(2)} \psi_{t}+\sum_{j=1}^{3} b_{j}^{(2)} \psi_{x_{j}}=0,
$$

$\widetilde{\mathrm{A}} \frac{1}{4}$ where

$$
\begin{gathered}
A^{(2)}=\left(\begin{array}{cc}
\bar{\chi} & 0 \\
0 & \bar{\sigma} I_{3}
\end{array}\right), \quad B_{i j}^{(2)}=\left(\begin{array}{cc}
\bar{\chi} & 0 \\
0 & \bar{\eta} I_{3} \delta_{i j}+\left(\bar{\zeta}+\frac{1}{3} \bar{\eta}\right)\left(e_{i} \otimes e_{j}+e_{j} \otimes e_{i}\right)
\end{array}\right), \\
a^{(2)}=\left(\begin{array}{cc}
c_{s}^{-2} & 0 \\
0 & I_{3}
\end{array}\right), \quad b_{j}=\left(e_{j} \otimes e_{0}+e_{0} \otimes e_{j}\right) .
\end{gathered}
$$

Finally, multiplying (3.2) by $\left(A^{(2)}\right)^{-\frac{1}{2}}$ and writting it in variables $\left(A^{(2)}\right)^{\frac{1}{2}} \psi$ gives

$$
\psi_{t t}-\sum_{i, j=1}^{3} \bar{B}_{i j} \psi_{x_{i} x_{j}}+a \psi_{t}+\sum_{j=1}^{3} b_{j} \psi_{x_{j}}=0,
$$

where

$$
\bar{B}_{i j}=\left(\begin{array}{cc}
1 & 0 \\
0 & \bar{\sigma}^{-1}\left(\bar{\eta} I_{3} \delta_{i j}+\left(\bar{\zeta}+\frac{1}{3} \bar{\eta}\right)\left(e_{i} \otimes e_{j}+e_{j} \otimes e_{i}\right)\right)
\end{array}\right)
$$




$$
a=\left(\begin{array}{cc}
c_{s}^{-2} \bar{\chi}^{-1} & 0 \\
0 & \bar{\sigma}^{-1} I_{3}
\end{array}\right), \quad b_{j}=(\bar{\chi} \bar{\sigma})^{-\frac{1}{2}}\left(e_{j} \otimes e_{0}+e_{0} \otimes e_{j}\right) .
$$

The goal is to prove a decay estimate for the Cauchy problem associated with (3.3):

$$
\begin{aligned}
\psi_{t t}-\sum_{i, j=1}^{3} B_{i j} \psi_{x_{i} x_{j}}+a \psi_{t}+\sum_{j=1}^{3} b_{j} \psi_{x_{j}} & =0 \text { on }(0, T] \times \mathbb{R}^{3}, \\
\psi(0) & ={ }^{0} \psi \text { on } \mathbb{R}^{3}, \\
\psi_{t}(0) & ={ }^{1} \psi \text { on } \mathbb{R}^{3} .
\end{aligned}
$$

3.1 Proposition. For some $s \in \mathbb{N}_{0}$ let $\left({ }^{0} \psi,{ }^{1} \psi\right) \in\left(H^{s+1} \times H^{s}\right) \cap\left(L^{1}\right)^{2}$ and $\psi(t) \in H^{s+1} \times H^{s}$ be a solution of (3.4)-(3.6). Then there exist $c, C>0$ such that for all integers $0 \leq k \leq s$ and all $t \in[0, T]$

$$
\begin{aligned}
\left\|\partial_{x}^{k} \psi(t)\right\|_{1}+\left\|\partial_{x}^{k} \psi_{t}(t)\right\| \leq C(1+t)^{-\frac{3}{4}-\frac{k}{2}}\left(\left\|{ }^{0} \psi\right\|_{L^{1}}+\left\|{ }^{1} \psi\right\|_{L^{1}}\right) \\
+C e^{-c t}\left(\left\|\partial_{x}^{k}\left({ }^{0} \psi\right)\right\|_{1}+\left\|\partial_{x}^{k}\left({ }^{1} \psi\right)\right\|\right) .
\end{aligned}
$$

To prove Proposition 3.1 we consider (3.4)-(3.6) in Fourier space, i.e.

$$
\begin{aligned}
\hat{\psi}_{t t}+|\xi|^{2} B(\check{\xi}) \hat{\psi}+a \hat{\psi}_{t}-i|\xi| b(\check{\xi}) \hat{\psi} & =0 \text { on }(0, T] \times \mathbb{R}^{3}, \\
\hat{\psi}(0) & ={ }^{0} \hat{\psi}(\xi) \text { on } \mathbb{R}^{3}, \\
\hat{\psi}_{t}(0) & ={ }^{1} \hat{\psi}(\xi) \text { on } \mathbb{R}^{3},
\end{aligned}
$$

where $\check{\xi}=\xi /|\xi|$,

$$
\begin{gathered}
B(\omega)=\sum_{i, j=1}^{3} \omega_{i} \bar{B}_{i j} \omega_{j}=\left(\begin{array}{cc}
1 & \\
0 & \bar{\sigma}^{-1}\left(\bar{\eta} I_{3}+\left(\bar{\zeta}+\frac{1}{3} \bar{\eta}\right)(\omega \otimes \omega)\right)
\end{array}\right), \\
b(\omega)=\sum_{j=1}^{3} b_{j} \omega_{j}=\left(\begin{array}{cc}
0 & \omega^{t} \\
\omega & 0
\end{array}\right), \omega \in \mathbb{S}^{2} .
\end{gathered}
$$

We get the following pointwise decay estimate.

3.2 Lemma. In the situation of Proposition 3.1 there exist $c, C>0$ such that for $(t, \xi) \in[0, T] \times \mathbb{R}^{n}$

$$
\begin{aligned}
& \left(1+|\xi|^{2}\right)|\hat{\psi}(t, \xi)|^{2}+\left|\hat{\psi}_{t}(t, \xi)\right|^{2} \\
& \leq C \exp (-c \rho(\xi) t)\left(\left.\left.\left(1+|\xi|^{2}\right)\right|^{0} \hat{\psi}(\xi)\right|^{2}+\left.\left.\right|^{1} \hat{\psi}(\xi)\right|^{2}\right),
\end{aligned}
$$

where $\rho(\xi)=|\xi|^{2} /\left(1+|\xi|^{2}\right)$. 
Proof. Our goal is to arrive at an expression of the form

$$
\frac{1}{2} \frac{d}{d t} E(t, \xi)+F(t, \xi) \leq 0,
$$

where $E(t, \xi)$ is uniformly equivalent to

$$
E_{0}(t, \xi)=(1+|\xi|)^{2}|\hat{\psi}(t, \xi)|^{2}+\left|\hat{\psi}_{t}(t, \xi)\right|^{2}
$$

and $F \geq c \rho(\xi) E_{0}$. Then (3.11) follows by Gronwall's Lemma.

W.l.o.g. assume $\xi=(|\xi|, 0,0)$ (otherwise rotate the coordinate system). Since $4 / 3 \bar{\eta}+\bar{\zeta}=\bar{\sigma},(3.8)$ decomposes into the two uncoupled systems

$$
\begin{array}{r}
w_{t t}+|\xi|^{2} w+\tilde{a} w-i|\xi| \tilde{b} w=0, \\
v_{t t}+\bar{\eta} \bar{\sigma}^{-1}|\xi|^{2} v+\bar{\sigma}^{-1} v_{t}=0,
\end{array}
$$

where $w=\left(\hat{\psi}_{0}, \hat{\psi}_{1}\right), v=\left(\hat{\psi}_{2}, \hat{\psi}_{3}\right)$,

$$
\tilde{a}=\left(\begin{array}{cc}
\bar{\chi}^{-1} c_{s}^{-2} & 0 \\
0 & \bar{\sigma}^{-1}
\end{array}\right), \quad \tilde{b}=(\bar{\chi} \bar{\sigma})^{-\frac{1}{2}}\left(\begin{array}{cc}
0 & 1 \\
1 & 0
\end{array}\right) .
$$

Obviously, this allows us to prove estimate (3.11) for $w$ and $v$ independently.

First, consider (3.14), where the estimate is fairly easy to obtain. Take the scalar product (in $\left.\mathbb{C}^{2}\right)$ of this equations with $v_{t}+1 /(2 \bar{\sigma}) v$. The real part reads

$$
\frac{1}{2} \frac{d}{d t} E^{(2)}+F^{(2)}=0
$$

where

$$
E^{(2)}=\left|v_{t}\right|^{2}+\frac{\bar{\eta}}{\bar{\sigma}}|\xi|^{2}|v|^{2}+\frac{1}{2 \bar{\sigma}^{2}}|v|^{2}+\frac{1}{\bar{\sigma}} \Re\left\langle v_{t}, v\right\rangle,
$$

and

$$
F^{(2)}=\frac{1}{2 \bar{\sigma}}\left|v_{t}\right|^{2}+\frac{\bar{\eta}}{2 \bar{\sigma}^{2}}|\xi|^{2}|v|^{2} .
$$

Since

$$
\left|\bar{\sigma}^{-1} \Re\left\langle v_{t}, v\right\rangle\right| \leq \frac{1}{3 \bar{\sigma}^{2}}|v|^{2}+\frac{3}{4}\left|v_{t}\right|^{2}
$$

$E^{(2)}$ is uniformly equivalent to $E_{0}^{(2)}=\left|v_{t}\right|^{2}+\left(1+|\xi|^{2}\right)|v|^{2}$ and as

$$
|\xi|^{2} \geq \frac{1}{2} \rho(\xi)\left(1+|\xi|^{2}\right)
$$

$F^{(2)} \geq c_{1} \rho(\xi) E_{0}^{(2)}$ for some $c_{1}>0$. 
Next, we study system (3.13). For notational purposes set $a_{1}=\bar{\chi}^{-1} c_{s}^{-1}$, $a_{2}=\bar{\sigma}^{-2}$ and $b_{1}=(\bar{\chi} \bar{\sigma})^{-\frac{1}{2}}$. Now, take the scalar product of (3.13) with $\tilde{a} w_{t}$. The real part of the resulting equation reads

$$
\frac{1}{2} \frac{d}{d t}\left(\left\langle\tilde{a} w_{t}, w_{t}\right\rangle+|\xi|^{2}\langle\tilde{a} w, w\rangle\right)+\left|\tilde{a} w_{t}\right|^{2}+\Re\left\langle-i|\xi| \tilde{b} w, \tilde{a} w_{t}\right\rangle=0 .
$$

Taking the scalar product of (3.13) with $-i|\xi| \tilde{b} w$ and considering the real part gives

$$
\frac{d}{d t}\left(\Re\left\langle w_{t},-i|\xi| \tilde{b} w\right\rangle\right)+\Re\left\langle\tilde{a} w_{t},-i|\xi| \tilde{b} w\right\rangle+|\xi|^{2}|\tilde{b} w|^{2}=0 .
$$

Then we take the scalar product of (3.13) with $w$. The real part is

$$
\frac{1}{2} \frac{d}{d t}\left(\langle a w, w\rangle+2 \Re\left\langle w_{t}, w\right\rangle\right)-\left|w_{t}\right|^{2}+|\xi|^{2}|w|^{2}=0 .
$$

Set

$$
S=\frac{1}{2 b_{1}}\left(\begin{array}{cc}
0 & a_{1}-a_{2} \\
a_{2}-a_{1} & 0
\end{array}\right) .
$$

Since $i S$ is hermitic

$$
\Re\left\langle i S w, w_{t}\right\rangle=\frac{1}{2} \frac{d}{d t}\langle i S w, w\rangle
$$

and we can write $(3.20)$ as

$$
\begin{aligned}
\frac{1}{2} \frac{d}{d t}\left(\langle a w, w\rangle+2 \Re\left\langle w_{t}, w\right\rangle+2|\xi|\langle i S w, w\rangle\right) & \\
& -\left|w_{t}\right|^{2}+|\xi|^{2}|w|^{2}-2 \Re\left(|\xi|\left\langle i S w, w_{t}\right\rangle\right)=0
\end{aligned}
$$

Now, add (3.18) $+(3.19)+\alpha(3.21)$ (for some $\alpha>0$ to be determined later) to obtain

$$
\frac{1}{2} \frac{d}{d t} E^{(1)}+F^{(1)}=0
$$

where

$$
\begin{aligned}
E^{(1)}=\left\langle\tilde{a} w_{t}, w_{t}\right\rangle+|\xi|^{2}\langle\tilde{a} w, w\rangle & +2 \Re\left(\left\langle w_{t},-i|\xi| \tilde{b} w\right\rangle\right) \\
& +\alpha\left(\langle\tilde{a} w, w\rangle+2 \Re\left\langle w_{t}, w\right\rangle+2|\xi|\langle i S w, w\rangle\right)
\end{aligned}
$$

and

$$
F^{(1)}=\left|\tilde{a} w_{t}\right|^{2}-\alpha\left|w_{t}\right|^{2}-2 \Re\left(i|\xi|\left\langle(\tilde{a} \tilde{b}-S) w, w_{t}\right\rangle\right)+|\xi|^{2}|\tilde{b} w|^{2}+\alpha|w|^{2} .
$$


First, show that $E^{(1)}$ is uniformly equivalent to $E_{0}^{(1)}=\left(1+|\xi|^{2}\right)|w|^{2}+\left|w_{t}\right|^{2}$. Obviously, there exists $C_{1}>0$ such that

$$
E^{(1)} \leq C_{1} E_{0}^{(1)} .
$$

For

$$
M=\left(\begin{array}{ll}
\tilde{a} & \tilde{b} \\
\tilde{b} & \tilde{a}
\end{array}\right)
$$

and $W=\left(w_{t},-i|\xi| w\right)$

$$
\left\langle\tilde{a} w_{t}, w_{t}\right\rangle+|\xi|^{2}\langle\tilde{a} w, w\rangle+2 \Re\left(\left\langle w_{t},-i|\xi| \tilde{b} w\right\rangle\right)=\langle M W, W\rangle_{\mathbb{C}^{4}} .
$$

It is easy to show that $\sigma(M)=\sigma(\tilde{a}+\tilde{b}) \cup \sigma(\tilde{a}-\tilde{b})$. Furthermore $c_{s} \in(0,1)$ yields $\tilde{a}+\tilde{b}>0, \tilde{a}-\tilde{b}>0$. Thus $M$ is positive definite, i.e.

$$
\left\langle\tilde{a} w_{t}, w_{t}\right\rangle+|\xi|^{2}\langle\tilde{a} w, w\rangle+2 \Re\left(\left\langle w_{t},-i|\xi| \tilde{b} w\right\rangle\right) \geq C_{2}\left(\left|w_{t}\right|^{2}+|\xi|^{2}|w|^{2}\right)
$$

for a $C_{2}>0$. Furthermore, by Young's inequality there exists $C_{3}>0$ such that

$$
\left|2 \Re\left\langle w_{t}, w\right\rangle+2 i\right| \xi|\langle S w, w\rangle| \leq \frac{d}{2}|w|^{2}+C_{3}\left(|\xi|^{2}|w|^{2}+\left|w_{t}\right|^{2}\right),
$$

where $d=\min \left\{a_{1}, a_{2}\right\}$. In conclusion

$$
E^{(1)} \geq C_{2}\left(\left|w_{t}\right|^{2}+|\xi|^{2}|w|^{2}\right)-\alpha C_{3}\left(|\xi|^{2}|w|^{2}+\left|w_{t}\right|^{2}\right)+\alpha \frac{d}{2}|w|^{2} .
$$

Hence, for $\alpha$ sufficiently small there exists $C_{4}>0$ such that

$$
E^{(1)} \geq C_{4} E_{0}^{(1)} .
$$

Finally show $F^{(1)} \geq c \rho(\xi) E_{0}^{(1)}$ for $\alpha$ sufficiently small. To this end write $F^{(1)}=F_{1}^{(1)}+F_{2}^{(1)}$, where

$$
\begin{aligned}
F_{1}^{(1)}= & \left(a_{1}^{2}-\alpha\right)\left|w_{t}^{1}\right|^{2}+\left(b_{1}^{2}+\alpha\right)|\xi|^{2}\left|w^{2}\right|^{2} \\
& -2 \Re\left(i|\xi|\left(a_{1} b_{1}+\alpha \frac{a_{1}-a_{2}}{2 b_{1}}\right) w^{2} \bar{w}_{t}^{1}\right), \\
F_{2}^{(1)}= & \left(a_{2}^{2}-\alpha\right)\left|w_{t}^{2}\right|^{2}+\left(b_{1}^{2}+\alpha\right)|\xi|^{2}\left|w^{1}\right|^{2} \\
& -2 \Re\left(i|\xi|\left(a_{2} b_{1}+\alpha \frac{a_{2}-a_{1}}{2 b_{1}}\right) w^{1} \bar{w}_{t}^{2}\right) .
\end{aligned}
$$


Since

$$
\left(a_{1}^{2}-\alpha\right)\left(b_{1}^{2}+\alpha\right)-\left(a_{1} b_{1}+\alpha \frac{a_{1}-a_{2}}{2 b_{1}}\right)^{2}=\alpha\left(a_{1} a_{2}-b_{1}^{2}\right)+O\left(\alpha^{2}\right)
$$

and $a_{1} a_{2}>b_{1}^{2}$ there exist $c_{2}>0$ such that

$$
F_{1}^{(1)} \geq \alpha c_{2}\left(\left|w_{t}^{1}\right|^{2}+|\xi|^{2}\left|w^{2}\right|^{2}\right)
$$

for $\alpha$ sufficiently small. In the same way we get

$$
F_{2}^{(1)} \geq \alpha c_{2}\left(\left|w_{t}^{2}\right|^{2}+|\xi|^{2}\left|w^{1}\right|^{2}\right) .
$$

Therefore

$$
F^{(1)} \geq \alpha c_{2}\left(\left|w_{t}\right|^{2}+|\xi|^{2}|w|^{2}\right) \geq \alpha \frac{c_{1}}{2} \rho(\xi) E_{0}^{(1)},
$$

which finishes the proof.

Based on Lemma 3.2 the proof for Proposition 3.1 goes as [1, Proof of Theorem 3.1].

Next consider the inhomogeneous initial-value problem

$$
\begin{aligned}
\psi_{t t}-\sum_{i, j=1}^{3} B_{i j} \psi_{x_{i} x_{j}}+a \psi_{t}-\sum_{j=1}^{n} b_{j} \psi_{x_{j}} & =h, \text { on }(0, T] \times \mathbb{R}^{3}, \\
\psi(0) & =\psi_{0}, \text { on } \mathbb{R}^{3} \\
\psi_{t}(0) & =\psi_{1} \text {, on } \mathbb{R}^{3} .
\end{aligned}
$$

for some $h:[0, T] \times \mathbb{R}^{3} \rightarrow \mathbb{R}^{4}$. We get the following results:

3.3 Proposition. Let $s$ be a non-negative integer,

$\left({ }^{0} \psi,{ }^{1} \psi\right) \in\left(H^{s+1} \times H^{s}\right) \cap\left(L^{1}\right)^{2}$ and $h \in C\left([0, T], H^{s} \cap L^{1}\right)$. Then the solution $\psi$ of (3.23)-(3.25) satisfies

$$
\begin{array}{r}
\left\|\partial_{x}^{k} \psi(t)\right\|_{1}+\left\|\partial_{x}^{k} \psi_{t}(t)\right\| \leq C(1+t)^{-\frac{3}{4}-\frac{k}{2}}\left(\left\|{ }^{0} \psi\right\|_{L^{1}}+\left\|{ }^{1} \psi\right\|_{L^{1}}\right) \\
+C e^{-c t}\left(\left\|\partial_{x}^{k}\left({ }^{0} \psi\right)\right\|_{1}+\left\|\partial_{x}^{k}\left({ }^{1} \psi\right)\right\|\right. \\
+C \int_{0}^{t}(1+t-\tau)^{-3 / 4-k / 2}\|h(\tau)\|_{L^{1}} \\
+C \exp (-c(t-\tau))\left\|\partial_{x}^{k} h(\tau)\right\| d \tau
\end{array}
$$

for all $t \in[0, T]$ and $0 \leq k \leq s$. 
Proof. For $t \in[0, T]$ let $T(t)$ be the linear operator which maps $\left({ }^{0} \psi,{ }^{1} \psi\right)$ to the solution $\left.(\psi(t)), \psi_{t}(t)\right)$ of the homogeneous IVP (3.4)-(3.6) at time $t$. By Duhamel's principle the solution of (3.23)-(3.25) is given by

$$
\left(\psi(t), \psi_{t}(t)\right)=T(t)\left({ }^{0} \psi,{ }^{1} \psi\right)+\int_{0}^{t} T(t-\tau)(0, h(\tau)) d \tau .
$$

Hence the assertion is an immediate consequence of Proposition 3.1

3.4 Proposition. Let $s$ be a non-negative integer. There exist $C_{1}, C_{2}>0$ such that for all $\left({ }^{0} \psi,{ }^{1} \psi\right) \in H^{s+1} \times H^{s}$ and $h \in C\left([0, T], H^{s}\right)$ the solution $\psi$ of (3.23)-(3.25) satisfies

$$
\begin{gathered}
C_{1}\left(\left\|\partial_{x}^{\alpha} \psi(t)\right\|_{1}^{2}+\left\|\partial_{x}^{\alpha} \psi_{t}(t)\right\|^{2}\right)+C_{1} \int_{0}^{t}\left\|\partial_{x}^{\alpha} \partial_{x} \psi(\tau)\right\|^{2}+\left\|\partial_{x}^{\alpha} \psi_{t}(\tau)\right\|^{2} d \tau \\
\leq C_{2}\left(\left\|\partial_{x}^{\alpha}\left({ }^{0} \psi\right)\right\|_{1}^{2}+\left\|\partial_{x}^{\alpha}\left({ }^{1} \psi\right)\right\|^{2}\right) \\
+\int_{0}^{t} C_{2}\left\|\partial_{x}^{\alpha} \psi(\tau)\right\|^{2}+\left(\partial_{x}^{\alpha} h(\tau), \frac{a}{2} \partial_{x}^{\alpha} \psi(\tau)+\partial_{x}^{\alpha} \psi_{t}(\tau)\right)_{L^{2}} d \tau \quad
\end{gathered}
$$

for all $t \in[0, T]$ and $\alpha \in \mathbb{N}_{0}^{3},|\alpha|=s$

Proof. Consider (3.23) in Fourier space, i.e.

$$
\hat{\psi}_{t t}+|\xi|^{2} B(\check{\xi}) \hat{\psi}+a \hat{\psi}_{t}-i|\xi| b(\check{\xi}) \hat{\psi}=\hat{h}
$$

We proceed similar as in the proof of Lemma 3.2. Again w.l.o.g. assume $\xi=(|\xi|, 0,0)$ then $(3.23)$ reads

$$
\begin{aligned}
w_{t t}+|\xi|^{2} w+\tilde{a} w-i|\xi| \tilde{b} w & =\left(\hat{h}^{0}, \hat{h}^{1}\right)^{t}, \\
v_{t t}+\bar{\eta} \bar{\sigma}^{-1}|\xi|^{2} v+\bar{\sigma}^{-1} v_{t} & =\left(\hat{h}^{2}, \hat{h}^{3}\right)^{t},
\end{aligned}
$$

where $w=\left(\hat{\psi}_{0}, \hat{\psi}_{1}\right), v=\left(\hat{\psi}_{2}, \hat{\psi}_{3}\right), \tilde{a}, \tilde{b}$ are given by (3.15). First, take the scalar product of (3.29) with $v_{t}+1 /(2 \bar{\sigma}) v$ and consider the real part

$$
\frac{1}{2} \frac{d}{d t} E^{(2)}+F^{(2)}=\Re\left\langle\left(\hat{h}^{2}, \hat{h}^{3}\right)^{t}, v_{t}+\frac{1}{2 \bar{\sigma}} v\right\rangle
$$

where $E^{(2)}, F^{(2)}$ are given by (3.16), (3.17). Since $E^{(2)}$ is uniformly equivalent to $\left|v_{t}\right|^{2}+\left(1+|\xi|^{2}\right)|v|^{2}$ and $F^{2} \geq c\left(\left|v_{t}\right|^{2}+|\xi|^{2}|v|^{2}\right)$ integrating (3.30) leads to

$$
\begin{aligned}
& C_{1}\left(\left|v_{t}\right|^{2}+\left(1+|\xi|^{2}\right)|v|^{2}\right)+C_{1} \int_{0}^{t}\left|v_{t}\right|^{2}+|\xi|^{2}|v|^{2} d \tau \\
& \quad \leq C_{2}\left(|v(0)|^{2}+\left(1+|\xi|^{2}\right)\left|v_{t}(0)\right|^{2}\right)+\int_{0}^{t} \Re\left\langle\left(\hat{h}^{2}, \hat{h}^{3}\right)^{t}, v_{t}+\frac{1}{2 \bar{\sigma}} v\right\rangle d \tau .
\end{aligned}
$$


Next, take the scalar product of (3.29) with $w_{t}+\tilde{a} / 2 w$. The real part reads

$$
\frac{1}{2} \frac{d}{d t} E^{(1)}+F^{(1)}=\Re\left\langle\left(\hat{h}^{0}, \hat{h}^{1}\right)^{t}, w_{t}+\frac{1}{2} \tilde{a} v\right\rangle,
$$

where

$$
E^{(1)}=\left|w_{t}\right|^{2}+|\xi|^{2}|w|^{2}+\frac{1}{2}|\tilde{a} w|^{2}+2 \Re\left\langle\tilde{a} w_{t}, w\right\rangle
$$

and

$$
F^{(1)}=\frac{1}{2}\left\langle\tilde{a} w_{t}, w_{t}\right\rangle+\Re\left\langle-i|\xi| \tilde{b} w, w_{t}\right\rangle+\frac{1}{2}|\xi|^{2}\langle\tilde{a} w, w\rangle-\frac{1}{2} \Re\langle i|\xi| \tilde{b} w, \tilde{a} w\rangle .
$$

Using Young's inequality it is easy to see that $E^{(1)}$ is uniformly equivalent to $\left|w_{t}\right|^{2}+\left(1+|\xi|^{2}\right)|w|^{2}$. Furthermore

$$
F^{(1)}=\frac{1}{2}\langle M W, W\rangle_{\mathbb{C}^{4}}-\frac{1}{2} \Re\langle i|\xi| \tilde{b} w, \tilde{a} w\rangle,
$$

where

$$
M=\left(\begin{array}{ll}
\tilde{a} & \tilde{b} \\
\tilde{b} & \tilde{a}
\end{array}\right)
$$

and $W=\left(w_{t},-i|\xi| w\right)$. As $M$ is positive definite (see proof of Lemma 3.2) there exists $c_{1}, c_{2}>0$ such that

$$
F^{(1)} \geq c_{1}\left(\left|w_{t}\right|^{2}+|\xi|^{2}|w|^{2}\right)-c_{2}|\xi||w||w| \geq \frac{c_{1}}{2}\left(\left|w_{t}\right|^{2}+|\xi|^{2}|w|^{2}\right)-\frac{c_{2}^{2}}{2 c_{1}}|w|^{2} .
$$

Thus integrating (3.32) leads to

$$
\begin{aligned}
& C_{1}\left(\left|w_{t}\right|^{2}+\left(1+|\xi|^{2}\right)|w|^{2}\right)+C_{1} \int_{0}^{t}\left|w_{t}\right|^{2}+|\xi|^{2}|w|^{2} d \tau \\
\leq & C_{2}\left(|w(0)|^{2}+\left(1+|\xi|^{2}\right)\left|w_{t}(0)\right|^{2}\right)+\int_{0}^{t} C_{2}|w|^{2}+\Re\left\langle\left(\hat{h}^{0}, \hat{h}^{1}\right)^{t}, w_{t}+\frac{\tilde{a}}{2} w\right\rangle d \tau .
\end{aligned}
$$

Adding (3.31) and (3.33) gives

$$
\begin{aligned}
& C_{1}\left(\left|\hat{\psi}_{t}\right|^{2}+\left(1+|\xi|^{2}\right)|\hat{\psi}|^{2}\right)+C_{1} \int_{0}^{t}\left|\hat{\psi}_{t}\right|^{2}+|\xi|^{2}|\hat{\psi}|^{2} d \tau \\
& \leq C_{2}\left(\left.\left.\right|^{0} \hat{\psi}\right|^{2}+\left.\left.\left(1+|\xi|^{2}\right)\right|^{1} \hat{\psi}\right|^{2}\right)+\int_{0}^{t} C_{2}|\hat{\psi}|^{2}+\Re\left\langle\hat{h}, \hat{\psi}_{t}+\frac{a}{2} \hat{\psi}\right\rangle d \tau
\end{aligned}
$$

Finally the assertion follows by multiplying (3.34) with $\xi^{2 \alpha}$ for $\alpha \in \mathbb{N}_{0}^{n}$, $|\alpha|=s$, integrating with respect to $\xi$ and using Plancherel's identity. 


\section{Global Existence and Asymptotic Decay of Small Solutions}

The goal of this section is to prove Theorem 2.1. We will proceed as follows: First we show a decay estimate for all but the highest order derivatives of a solution, proposition 4.1, and then an energy estimate for the derivatives of highest order, proposition 4.3. Then Theorem 2.1 follows from combining the two, proposition 4.4.

As in section 3. fix $\theta>0$, multiply (2.2) by $(n s)^{-1} \theta^{-2}\left(A^{(2)}\right)^{-\frac{1}{2}}$ and change the variables to $\left(A^{(2)}\right)^{\frac{1}{2}} \psi$ such that the linearisation at $\left(\theta^{-1}, 0,0,0\right)$ is given by (3.3). In addition consider $\psi-\bar{\psi}$ with $\bar{\psi}=\left(\theta^{-1}, 0,0,0\right)$ instead of $\psi, A(\cdot+\bar{\psi})$ instead of $A(\cdot)$ and so on, such that the rest state is shifted from $\left(\theta^{-1}, 0,0,0\right)$ to $(0,0,0,0)$. Furthermore write $U=\left(\psi, \psi_{t}\right)$ and $U_{0}=\left({ }^{0} \psi,{ }^{1} \psi\right)$ for a solution to (2.3)-(2.5) and the initial values, respectively.

Throughout this section let $s \geq s_{0}+1\left(s_{0}=[3 / 2]+1\right), T>0, U_{0} \in$ $H^{s+1} \times H^{s}$, and $\psi$ satisfy

$$
\psi \in \bigcap_{j=0}^{s} C^{j}\left([0, T], H^{s+1-j}\right)
$$

For $0 \leq t \leq t_{1} \leq T$ define

$$
N_{s}\left(t, t_{1}\right)^{2}=\sup _{\tau \in\left[t, t_{1}\right]}\|U(\tau)\|_{s+1, s}^{2}+\int_{t}^{t_{1}}\|U(\tau)\|_{s+1, s}^{2} d \tau
$$

We write $N_{s}(t)$ instead of $N_{s}(0, t)$. Furthermore assume that $N_{s}(T) \leq a_{0}$ for an $a_{0}>0$. Since $s \geq s_{0}, H^{s} \hookrightarrow L^{\infty}$ is a continuous embedding. Hence $N_{s}(T) \leq a_{0}$ implies that $\left(\psi, \psi_{t}, \partial_{x} \psi\right)$ takes values in a closed ball $\overline{B(0, r)} \subset \mathbb{R}^{4} \times \mathbb{R}^{4} \times \mathbb{R}^{12}$ for some $r>0$.

First we prove the decay estimate. To this end it is convenient to rewrite (the modified version of) (2.3) as

$$
\psi_{t t}-\sum_{i, j=1}^{3} \bar{B}_{i j} \psi_{x_{i} x_{j}}+a \psi_{t}+\sum_{j=1}^{3} b_{j} \psi_{x_{j}}=h\left(\psi, \psi_{t}, \partial_{x} \psi, \partial_{x}^{2} \psi\right),
$$


where

$$
\begin{aligned}
h\left(\psi, \psi_{t}, \partial_{x} \psi, \partial_{x}^{2} u\right)= & \sum_{i, j=1}^{3}\left(A(\psi)^{-1} B_{i j}(\psi)-\bar{B}_{i j}\right) \psi_{x_{i} x_{j}} \\
& +\sum_{j=1}^{3} A(\psi)^{-1} D_{j}(\psi) \psi_{t x_{j}} \\
& -A(\psi)^{-1} f\left(\psi, \psi_{t}, \partial_{x} \psi\right)+\left(a \psi_{t}+\sum_{j=1}^{3} b_{j} \psi_{x_{j}}\right) .
\end{aligned}
$$

4.1 Proposition. There exist constants $a_{1}\left(\leq a_{0}\right), \delta_{1}=\delta_{1}\left(a_{1}\right), C_{1}=$ $C_{1}\left(a_{1}, \delta_{1}\right)>0$ such that the following holds: If $N_{s}(T)^{2} \leq a_{1}$ and $\left\|U_{0}\right\|_{s, s-1,1}^{2} \leq \delta_{1}$ for a solution $\psi$ of (2.3)-(2.5) satisfying (4.1), then

$$
\|U(t)\|_{s, s-1} \leq C_{1}(1+t)^{-\frac{3}{4}}\left\|U_{0}\right\|_{s, s-1,1} \quad(t \in[0, T]) .
$$

Proof. Let $t \in[0, T]$ and $\psi$ be a solution to (2.3)-(2.5). Since $B_{i j}(0)=\bar{B}_{i j}$, $D_{j}(0)=0$ and

$$
a \psi_{t}+\sum_{j=1}^{3} b_{j} \psi_{x_{j}}=\operatorname{Df}(0)\left(\psi, \psi_{t}, \partial_{x} \psi\right)
$$

Lemmas A.1, A.2 show that there exists a $c>0\left(c \leq a_{0}\right)$ such that $h(t) \in H^{s-1} \cap L^{1}$ and

$$
\begin{aligned}
\|h(t)\|_{s-1} & \leq C\|\psi(t)\|_{s-1}\left(\left\|\partial_{x}^{2} \psi(t)\right\|_{s-1}+\left\|\partial_{x} \psi_{t}(t)\right\|_{s-1}\right) \\
& +C \|\left(\psi(t), \psi_{t}(t), \partial_{x} \psi(t) \|_{s-1}^{2}\right. \\
& \leq C\|U(t)\|_{s+1, s}\|U(t)\|_{s, s-1}, \\
\|h(t)\|_{L^{1}} & \leq C\|U(t)\|_{2,1}^{2},
\end{aligned}
$$

if $N_{s}(T) \leq c$, which we will assume throughout this proof. Then Proposition 3.3 yields

$$
\begin{aligned}
\|U(t)\|_{s, s-1} & \leq C(1+t)^{-\frac{3}{4}}\left\|U_{0}\right\|_{s, s-1,1} \\
+ & C \int_{0}^{t} \exp (-c(t-\tau))\|h(\tau)\|_{s-1}+(1+t-\tau)^{-\frac{3}{4}}\|h(\tau)\|_{L^{1}} d \tau
\end{aligned}
$$


This leads to

$$
\begin{aligned}
\|U(t)\|_{s-1, s} \leq C(1+t)^{-\frac{3}{4}}\left\|U_{0}\right\|_{s, s-1,1} & \\
+C \sup _{\tau \in[0, t]}\|U(\tau)\|_{s+1, s} \int_{0}^{t} & \exp (-c(t-\tau))\|U(\tau)\|_{s, s-1} d \tau \\
& \quad+C \int_{0}^{t}(1+t-\tau)^{-\frac{3}{4}}\|U(\tau)\|_{s, s-1}^{2} d \tau .
\end{aligned}
$$

Multiplying with $(1+t)^{\frac{3}{4}}$ gives

$$
\begin{aligned}
& (1+t)^{\frac{3}{4}}\|U(t)\|_{s, s-1} \leq C\left\|U_{0}\right\|_{s, s-1,1} \\
& +C N_{s}(t) \mu_{1}(t) \sup _{\tau \in[0, t]}(1+\tau)^{\frac{3}{4}}\|U(\tau)\|_{s, s-1} \\
& \quad+C \mu_{2}(t) \sup _{\tau \in[0, t]}(1+\tau)^{\frac{3}{2}}\|U(\tau)\|_{s, s-1}^{2},
\end{aligned}
$$

where

$$
\begin{aligned}
& \mu_{1}(t)=(1+t)^{\frac{3}{4}} \int_{0}^{t} \exp (-c(t-\tau))(1+\tau)^{-\frac{3}{4}} d \tau \\
& \mu_{2}(t)=(1+t)^{\frac{3}{4}} \int_{0}^{t}(1+t-\tau)^{-\frac{3}{4}}(1+\tau)^{-\frac{3}{2}} d \tau .
\end{aligned}
$$

Since $\mu_{1}, \mu_{2}$ are bounded functions on $[0, \infty)$, we get

$$
\begin{aligned}
\sup _{\tau \in[0, t]}(1+\tau)^{\frac{3}{4}}\|U(\tau)\|_{s, s-1} \leq C\left\|U_{0}\right\|_{s, s-1,1} & \\
+C N_{s}(t) \sup _{\tau \in[0, t]}(1+\tau)^{\frac{3}{4}}\|U(\tau)\|_{s, s-1} & \\
& +C \sup _{\tau \in[0, t]}(1+\tau)^{\frac{3}{2}}\|U(\tau)\|_{s, s-1}^{2} .
\end{aligned}
$$

We can deduce from this equation that there in fact exists $a_{1}>0\left(a_{1} \leq c\right)$, $\delta_{1}>0$ and $C_{1}>0$, such that

$$
\sup _{\tau \in[0, t]}(1+\tau)^{\frac{3}{4}}\|U(\tau)\|_{s, s-1} \leq C_{1}\left\|U_{0}\right\|_{s, s-1,1},
$$

whenever $N_{s}(T)^{2} \leq a_{1}$ and $\left\|U_{0}\right\|_{s, s-1,1}^{2} \leq \delta_{1}$. 
4.2 Corollary. In the situation of Proposition 4.1 there exists a $C_{2}=$ $C_{2}\left(a_{1}, \delta_{1}\right)>0$ such that

$$
N_{s-1}(T)^{2} \leq C_{2}\left\|U_{0}\right\|_{s, s-1,1}^{2}
$$

whenever $N_{s}(T)^{2} \leq a_{1}$ and $\left\|U_{0}\right\|_{s, s-1,1}^{2} \leq \delta_{1}$.

Proof. The function $t \mapsto(1+t)^{-\frac{3}{4}}$ is square-integrable on $[0, \infty)$. Therefore the assertion is a direct consequence of Proposition 4.1.

Now it is convenient to write (the modified version of) (2.3) as

$$
\psi_{t t}-\sum_{i, j=1}^{3} \bar{B}_{i j} \psi_{x_{i} x_{j}}+a \psi_{t}+\sum_{j=1}^{3} b_{j} \psi_{x_{j}}=L(\psi) \psi+h_{2}\left(\psi, \psi_{t}, \partial_{x} \psi\right)
$$

where

$$
\begin{aligned}
L(\psi) \psi= & (I-A(\psi)) \psi_{t t}+\sum_{i, j=1}^{3}\left(\bar{B}_{i j}-B_{i j}(\psi)\right) \psi_{x_{i} x_{j}}-\sum_{j=1}^{3} D_{j}(\psi) \psi_{t x_{j}}, \\
& h_{2}\left(\psi, \psi_{t}, \partial_{x} \psi\right)=a \psi_{t}+\sum_{j=1}^{3} b_{j} \psi_{x_{j}}-f\left(\psi, \psi_{t}, \partial_{x} \psi\right) .
\end{aligned}
$$

4.3 Proposition. There exist constants $a_{2}\left(\leq a_{0}\right)$ and $c_{3}, C_{3}=C_{3}\left(a_{2}\right)>0$ such that the following holds: If $N_{s}(T)^{2} \leq a_{2}$ for a solution $\psi$ of $(2.3)-(2.5)$ satisfying (4.1), then

$$
\begin{aligned}
& \left\|\partial_{x}^{s} \psi(t)\right\|_{1}^{2}+\left\|\partial_{x}^{s} \psi_{t}(t)\right\|^{2}+\int_{0}^{t}\left\|\partial_{x}^{s+1} \psi(\tau)\right\|^{2}+\left\|\partial_{x}^{s} \psi_{t}(\tau)\right\|^{2} d \tau \\
& \quad-c_{3} \int_{0}^{t}\left\|\partial_{x}^{s} \psi(\tau)\right\|^{2} d \tau \leq C_{3}\left(\left\|U_{0}\right\|_{s, s+1}^{2}+N_{s}(t)^{3}\right) \quad(t \in[0, T]) .
\end{aligned}
$$

Proof. We prove the result in two steps.

Step 1:

First let $U_{0}=\left({ }^{0} \psi,{ }^{1} \psi\right) \in H^{s+1} \times H^{s}$ and $\psi$ be a solution to (2.3)-(2.5) such that

$$
\psi \in \bigcap_{j=0}^{s} C^{j}\left([0, T], H^{s+2-j}\right) .
$$

By Lemma A.2 there exists a $c>0$ such that $I-A(\psi), \bar{B}_{i j}-B_{i j}(\psi), D_{j}(\psi) \in$ $H^{s+1}$ provided $N_{s}(T) \leq c$. We will assume this throughout the proof. Then 
due to (4.8) and [6, Lemma 2.3] $L(\psi) \psi \in H^{s}$. Lemma A.2 yields $h_{2} \in H^{s}$. Thus we can conlcude by Proposition 3.4 that

$$
\begin{gathered}
C_{1}\left(\left\|\partial_{x}^{\alpha} \psi(t)\right\|_{1}^{2}+\left\|\partial_{x}^{\alpha} \psi_{t}(t)\right\|^{2}\right)+C_{1} \int_{0}^{t}\left\|\partial_{x}^{\alpha} \partial_{x} \psi(\tau)\right\|^{2}+\left\|\partial_{x}^{\alpha} \psi_{t}(\tau)\right\|^{2} d \tau \\
\leq C_{2}\left(\left\|\partial_{x}^{\alpha}\left({ }^{0} \psi\right)\right\|_{1}^{2}+\left\|\partial_{x}^{\alpha}\left({ }^{1} \psi\right)\right\|^{2}\right) \\
+C_{2} \int_{0}^{t}\left\|\partial_{x}^{\alpha} \psi(\tau)\right\|^{2} d \tau \\
\int_{0}^{t}\left(\partial_{x}^{\alpha}\left(L(\psi(\tau)) \psi(\tau)+h_{2}(\tau)\right), \partial_{x}^{\alpha} \psi_{t}(\tau)+\frac{a}{2} \partial_{x}^{\alpha} \psi(\tau)\right)_{L^{2}} d \tau
\end{gathered}
$$

for all $\alpha \in \mathbb{N}_{0}^{3},|\alpha|=s$. First obviously

$$
\left|\left(\partial_{x}^{\alpha} h_{2}, \partial_{x}^{\alpha} \psi_{t}+\frac{a}{2} \partial_{x}^{\alpha} \psi\right)_{L^{2}}\right| \leq C\left\|h_{2}\right\|_{s}\|U\|_{s}
$$

and integrating by parts gives

$$
\begin{aligned}
\left|\left(\partial_{x}^{\alpha}(L(\psi) \psi), \frac{a}{2} \partial_{x}^{\alpha} \psi\right)_{L^{2}}\right| & \leq C\|L(\psi) \psi\|_{s-1}\|\psi\|_{s+1} \\
& \leq C\|I-A(\psi)\|\left\|_{s}\right\| \psi_{t t}\left\|_{s-1}\right\| \psi \|_{s+1} \\
& +C \sum_{i, j=1}^{3}\left\|\bar{B}_{i j}-B_{i j}(\psi)\right\|_{s}\left\|\partial_{x}^{2} \psi\right\|_{s-1}\|\psi\|_{s+1} \\
& +C \sum_{j=1}^{3}\left\|D_{j}(\psi)\right\|_{s}\left\|\partial_{x} \psi\right\|_{s-1}\|\psi\|_{s+1} .
\end{aligned}
$$

Next write

$$
\begin{aligned}
& \partial_{x}^{\alpha}(L(\psi) \psi)=L(\psi) \partial_{x}^{\alpha} \psi+\left[\partial_{x}^{\alpha},(I-A(\psi))\right] \psi_{t t} \\
& \quad+\sum_{i, j=1}^{n}\left[\partial_{x}^{\alpha},\left(\bar{B}_{i j}-B_{i j}(\psi)\right)\right] \psi_{x_{i} x_{j}}+\sum_{j=1}^{n}\left[\partial_{x}^{\alpha}, D_{j}(\psi)\right] \psi_{t x_{j}} .
\end{aligned}
$$

Since $I-A(\psi), \bar{B}_{i j}-B_{i j}(\psi), D_{j}(\psi) \in H^{s}$, [6, Lemma 2.5(i)] yields

$$
\begin{aligned}
\left\|\left[\partial_{x}^{\alpha},(-A(\psi))\right] \psi_{t t}\right\| & \leq C\left\|\partial_{x} A(\psi)\right\|_{s-1}\left\|\psi_{t t}\right\|_{s-1} \\
\left\|\left[\partial_{x}^{\alpha},\left(\bar{B}_{i j}-B_{i j}(\psi)\right)\right] \psi_{x_{i} x_{j}}\right\| & \leq C\left\|\partial_{x} B_{i j}(\psi)\right\|_{s-1}\left\|\psi_{x_{i} x_{j}}\right\|_{s-1} \\
\left\|\left[\partial_{x}^{\alpha}, D_{j}\right] \psi_{t x_{j}}\right\| & \leq C\left\|\partial_{x} D_{j}(\psi)\right\|_{s-1}\left\|\psi_{t x_{j}}\right\|_{s-1} .
\end{aligned}
$$


Furthermore integration by parts and the symmetry of $A, B_{i j}$ and $D_{j}$ yield

$$
\begin{gathered}
\int_{0}^{t}\left(L(\psi) \partial_{x}^{\alpha} \psi, \partial_{x}^{\alpha} \psi_{t}\right)_{L^{2}} d \tau \\
\leq C \int_{0}^{t}\left\|\partial_{t} A\right\|\left\|\partial_{x}^{\alpha}\left(\partial_{x} \psi, \psi_{t}\right)\right\|^{2} \\
\quad+\left(\sum_{i, j=1}^{n}\left\|\partial_{t} B_{i j}\right\|+\left\|\partial_{x} B_{i j}\right\|+\sum_{j=1}^{n}\left\|\partial_{x} D_{j}\right\|\right)\left\|\partial_{x}^{\alpha}\left(\partial_{x} \psi, \psi_{t}\right)\right\|^{2} d \tau \\
+C\left(\|I-A\|+\sum_{i, j=1}^{n}\left\|\bar{B}_{i j}-B_{i j}\right\|\right)\left\|\partial_{x}^{\alpha}\left(\partial_{x} \psi, \psi_{t}\right)\right\|^{2}+C\left\|\partial_{x}^{\alpha}\left(\partial_{x}{ }^{0} \psi,{ }^{1} \psi\right)\right\|^{2},
\end{gathered}
$$

In conclusion, (4.9) and the estimates (4.10), (4.11), (4.12) and (4.13) lead to

$$
\begin{array}{r}
\left\|\partial_{x}^{\alpha} \psi(t)\right\|_{1}^{2}+\left\|\partial_{x}^{\alpha} \psi_{t}(t)\right\|^{2}+\int_{0}^{t}\left\|\partial_{x}^{\alpha} \partial_{x} \psi(\tau)\right\|^{2}+\left\|\partial_{x}^{\alpha} \psi_{t}(\tau)\right\|^{2} d \tau \\
-c \int_{0}^{t}\left\|\partial_{x}^{\alpha} \psi(\tau)\right\|^{2} d \tau \\
\leq C\left\|U_{0}\right\|_{s+1, s}^{2}+C \int_{0}^{t}\left(\left\|h_{2}\right\|+R_{1}(\psi)\right)\|U\|_{s+1, s}^{2} d \tau \\
+C \int_{0}^{t}\|I-A(\psi)\|_{s}\left\|\psi_{t t}\right\|_{s-1}\|U\|_{s+1, s} d \tau \\
+C R_{2}(\psi)\|U(t)\|_{s+1, s}^{2}
\end{array}
$$

where

$$
\begin{array}{r}
R_{1}(\psi)=\left\|\partial_{t} A(\psi)\right\|+\|I-A(\psi)\|_{s}+\sum_{i, j=1}^{n}\left\|\partial_{t} B_{i j}(\psi)\right\|+ \\
+\bar{B}_{i j}-B_{i j}(\psi) \|_{s} \\
+\sum_{j=1}^{n}\left\|D_{j}(\psi)\right\|_{s}
\end{array}
$$

and

$$
R_{2}(\psi)=\|I-A(\psi)\|+\sum_{i, j=1}^{n}\left\|\bar{B}_{i j}-B_{i j}(\psi)\right\|
$$

\section{Step 2:}


Now let $\psi$ be a solution to (2.3)-(2.5) satisfying (4.1). For $\delta>0$ set $\psi^{\delta}=\phi_{\delta} * \psi$. Applying $\phi_{\delta} *$ to (4.6) yields

$$
\psi_{t t}^{\delta}-\sum_{i, j=1}^{3} \bar{B}_{i j} \psi^{\delta}+a \psi_{t}^{\delta}+\sum_{j=1}^{3} b_{j} \psi_{x_{j}}^{\delta}=L(\psi) \psi^{\delta}+R^{\delta}(\psi)+h_{2}^{\delta}
$$

where $h^{\delta}=\phi_{\delta} * h_{2}$ and

$$
\begin{aligned}
R^{\delta}(\psi)=\left[\phi_{\delta} *,(I-A(\psi))\right] \psi_{t t}+\sum_{i, j=1}^{n}\left[\phi_{\delta} *, \bar{B}_{i j}-B_{i j}(\psi)\right] \psi_{x_{i} x_{j}} & \\
& +\sum_{j=1}^{3}\left[\phi_{\delta}, D_{j}(\psi)\right] \psi_{t x_{j}} .
\end{aligned}
$$

Due to [6, Lemma 2.5 (ii)] $R^{\delta} \in H^{s}$. Hence $L(\psi) \psi^{\delta}+R^{\delta}+h_{2}^{\delta} \in H^{s}$. Thus proceeding as in step 1 yields

$$
\begin{gathered}
\left\|\partial_{x}^{\alpha} \psi^{\delta}(t)\right\|_{1}^{2}+\left\|\partial_{x}^{\alpha} \psi_{t}^{\delta}(t)\right\|^{2}+\int_{0}^{t}\left\|\partial_{x}^{\alpha} \partial_{x} \psi^{\delta}(\tau)\right\|^{2}+\left\|\partial_{x}^{\alpha} \psi_{t}^{\delta}(\tau)\right\|^{2} d \tau \\
-c \int_{0}^{t}\left\|\partial_{x}^{\alpha} \psi^{\delta}(\tau)\right\|^{2} d \tau \\
\leq C\left\|U_{0}^{\delta}\right\|_{s+1, s}^{2} \\
+C \int_{0}^{t}\left(\left\|h_{2}^{\delta}\right\|_{s}+R_{1}(\psi)\right)\left\|U^{\delta}\right\|_{s+1, s}^{2}+\|I-A(\psi)\|_{s}\left\|\psi_{t t}^{\delta}\right\|_{s-1}\left\|U^{\delta}\right\|_{s+1, s} d \tau \\
+C \int_{0}^{t}\left\|R^{\delta}(\psi)\right\|_{s}\left\|U^{\delta}\right\|_{s+1, s} d \tau+C R_{2}(\psi)\left\|U^{\delta}(t)\right\|_{s+1, s}^{2}
\end{gathered}
$$

It is easy to see that $U^{\delta} \rightarrow U$ and $h_{2}^{\delta} \rightarrow h_{2}$ in $L^{\infty}\left([0, T], H^{s+1} \times H^{s}\right)$ and in $L^{2}\left([0, T], H^{s}\right)$ respectively as $\delta \rightarrow 0$. Furthermore $R^{\delta}(\psi) \rightarrow 0$ in $L^{2}\left([0, T], H^{s}\right)$ as $\delta \rightarrow 0$ due to [6, Lemma 2.5(ii)]. Thus we get (4.14) for $\psi$ satisfying (4.1).

Furthermore by Lemmas A.1 and A.2

$$
\left\|h_{2}\right\|_{s}+R_{1}(\psi)+R_{2}(\psi) \leq C\|U\|_{s+1, s}^{2} .
$$

for $N_{s}(T)$ sufficiently small. Finally, since $\psi$ satisfies (2.3), we get

$$
\left\|\psi_{t t}\right\|_{s-1} \leq C\left(\left\|\partial_{x}^{2} \psi\right\|_{s-1}+\left\|f\left(\psi, \psi_{t}, \partial_{x} \psi\right)\right\|_{s-1}\right) \leq\|U\|_{s+1, s}
$$


for $N_{s}(T)$ sufficiently small. Hence we can deduce from (4.14) that

$$
\begin{gathered}
\left\|\partial_{x}^{\alpha} \psi(t)\right\|_{1}^{2}+\left\|\partial_{x}^{\alpha} \psi_{t}(t)\right\|^{2}+\int_{0}^{t}\left\|\partial_{x}^{\alpha} \partial_{x} \psi(\tau)\right\|^{2}+\left\|\partial_{x}^{\alpha} \psi_{t}(\tau)\right\|^{2} d \tau \\
\quad-c \int_{0}^{t}\left\|\partial_{x}^{\alpha} \psi(\tau)\right\|^{2} d \tau \\
\leq C\left\|U_{0}\right\|_{s+1, s}^{2}+C\|U(t)\|_{s+1, s}^{3}+C \int_{0}^{t}\|U(\tau)\|_{s+1, s}^{3} d \tau
\end{gathered}
$$

The assertion is an immediate consequence of this inequality.

4.4 Proposition. In the situation of Proposition 4.1 there exist constants $a_{3}\left(\leq \min \left\{a_{2}, a_{1}\right\}\right), C_{4}=C_{4}\left(a_{3}, \delta_{1}\right)>0$ ( $\delta_{1}$ being the constant in Proposition 4.1) such that the the following holds: If $N_{s}(T)^{2} \leq a_{3}$ and $\left\|U_{0}\right\|_{s, s-1,1}^{2} \leq \delta_{1}$ for a solution $\psi$ of (2.3)-(2.5) satisfying (4.1), then

$$
N_{s}(t)^{2} \leq C_{4}^{2}\left\|U_{0}\right\|_{s+1, s, 1}^{2} \quad(t \in[0, T])
$$

Proof. This follows directly by adding (4.5) $+\varepsilon(4.7)$ for $\varepsilon$ sufficiently small.

Finally we turn to the proof of Theorem 2.1.

Proof of Theorem 2.1. Let $T_{1}>0, \delta_{2}>0$ such that for all $U_{0}=\left(\psi_{0}, \psi_{1}\right) \in$ $H^{s+1} \times H^{s}$, where $\left\|U_{0}\right\|_{s+1, s}^{2}<\delta_{2}$, there exists a solution $U=\left(\psi, \psi_{t}\right)$ of the Cauchy problem (2.3)-(2.5) with

$$
\psi \in \bigcap_{j=1}^{s} C^{j}\left(\left[0, T_{1}\right], H^{s+1-j}\right) .
$$

This is possible due to [5, Theorem III]. Furthermore let $a_{3}, \delta_{1}$ and $C_{4}$ be the constants in Proposition 4.4. Choose $0<\varepsilon<a_{3} /\left(2\left(1+T_{1}\right)\right)$. Due to [5, Ibid.] there exists $\delta_{3}>0,\left(\delta_{3} \leq \delta_{2}\right)$ such that for all $U_{0}=\left(\psi_{0}, \psi_{1}\right) \in$ $H^{s+1} \times H^{s}$, where $\left\|U_{0}\right\|_{s+1, s}^{2}<\delta_{3}$ the solution $U$ of (2.3)-(2.5) satisfies

$$
\sup _{t \in\left[0, T_{1}\right]}\|U(t)\|_{s+1, s}^{2}<\varepsilon .
$$

Now set $\delta_{0}=\min \left\{\delta_{1}, \delta_{3}, \delta_{3} / C_{4}, a_{3} /\left(2 C_{4}\right)\right\}$ and choose any $U_{0} \in\left(H^{s+1} \times H^{s}\right) \cap$ $\left(L^{1} \times L^{1}\right)$ for which $\left\|U_{0}\right\|_{s+1, s, 1}^{2}<\delta_{0}$. Since $\delta_{0} \leq \delta_{3}$

$$
N_{s}\left(T_{1}\right)^{2}<\varepsilon+T_{1} \varepsilon<\frac{a_{3}}{2} .
$$


Hence by Proposition 4.4 and $\left\|U_{0}\right\|_{s+1, s, 1}^{2}<\delta_{1}$

$$
N_{s}\left(T_{1}\right)^{2} \leq C_{4}\left\|U_{0}\right\|_{s+1, s}^{2}<C_{4} \delta_{0} \leq \delta_{3} .
$$

Furthermore due to Proposition 4.1, (2.7) holds for all $t \in\left[0, T_{1}\right]$. In particular (4.16) yields

$$
\left\|U\left(T_{1}\right)\right\|_{s+1, s}^{2}<\delta_{3} .
$$

Thus we can solve $(2.3)$ on $\left[T_{1}, 2 T_{1}\right]$ with initial values $\left(\psi\left(T_{1}\right), \psi_{t}\left(T_{1}\right)\right)$ and get

$$
N_{s}\left(T_{1}, 2 T_{1}\right)^{2} \leq \varepsilon+T_{1} \varepsilon<\frac{a_{3}}{2} .
$$

Now extend the solution $\left(\psi, \psi_{t}\right)$ continuously on $\left[0,2 T_{1}\right]$. We can conclude

$$
N_{s}\left(2 T_{1}\right)^{2} \leq N_{s}\left(T_{1}\right)^{2}+N_{s}\left(T_{1}, 2 T_{1}\right)^{2}<\frac{a_{3}}{2}+\frac{a_{3}}{2}=a_{3} .
$$

Since we have already assumed $\left\|U_{0}\right\|_{s+1, s, 1}^{2}<\delta_{1}$ Propositions 4.4 and 4.1 yield

$$
N_{s}\left(2 T_{1}\right) \leq C_{4} \delta_{0}
$$

and (2.7) holds for all $t \in\left[0,2 T_{1}\right]$. Due to (4.18) we can repeat the former argument to obtain a solution on $\left[0,3 T_{1}\right]$ and further repetition proves the assertion.

\section{A. Appendix}

A.1 Lemma. Let $n, N \in \mathbb{N}, s \geq s_{0}:=\left[\frac{n}{2}\right]+1$ and $F \in C^{\infty}\left(\mathbb{R}^{N}\right), F(0)=0$. Then there exist $\delta, C=C(\delta)>0$ such that for all $u \in H^{s}$ with $\|u\|_{s} \leq \delta$, $F(u)-\partial_{u} F(0) \in H^{s}$ and

$$
\left\|F(u)-\partial_{u} F(0) u\right\|_{s} \leq C\|u\|_{s}^{2} .
$$

Proof. Since $s \geq s_{0}$ there exists a $C_{1}>0$ such that

$$
\|u\|_{L^{\infty}} \leq C_{1}\|u\|_{s}
$$

for all $u \in H^{s}$. Furthermore due to $F(0)=0$ there exist $\delta_{1}, C_{2}=C_{2}(\delta)>0$ such that

$$
\left|F(y)-\partial_{y} F(0) y\right| \leq C_{2}|y|^{2} .
$$

for all $y \in \mathbb{R}^{N}$ with $|y| \leq \delta_{1}$. Now let $u \in H^{s}$ such that $\|u\|_{s} \leq \delta_{1} / C_{1}$ (i.e. $\left.\|u\|_{L^{\infty}} \leq \delta_{1}\right)$. Then

$$
\left\|F(u)-\partial_{u} F(0) u\right\| \leq C_{2}\|u\|_{L^{\infty}}\|u\| \leq C_{1} C_{2}\|u\|_{s}^{2} .
$$


Furthermore for $\alpha \in \mathbb{N}_{0}^{n}$ with $1 \leq|\alpha|=j \leq s$ we get

$$
\partial_{x}^{\alpha} F(u)=\partial_{u} F(u) \partial_{x}^{\alpha} u+R,
$$

where

$$
R=\sum_{1 \leq|\beta|<j}\left(\begin{array}{l}
\alpha \\
\beta
\end{array}\right) \partial_{x}^{\beta} u \partial_{x}^{\alpha-\beta} F(u) .
$$

Since $\partial_{x} u \in H^{s-1}$ and $\|u\|_{L^{\infty}} \leq \delta_{1}, \partial_{x} F(u) \in H^{s-1}$ and

$$
\left\|\partial_{x} F(u)\right\|_{s-1} C_{3}\left\|\partial_{x} u\right\|_{s-1}
$$

for a $C_{3}=C_{3}\left(\delta_{2}\right)>0$ by [6, Lemma 2.4 ]. Therefore [6, Lemma 2.3] yields

$$
\|R\| \leq C_{4}\left\|\partial_{x} u\right\|_{s-1}\left\|\partial_{x} F(u)\right\|_{s-1} \leq C_{3} C_{4}\left\|\partial_{x} u\right\|_{s-1}^{2}
$$

for a $C_{4}>0$. On the other hand there exist $\delta_{2}, C_{5}=C_{5}\left(\delta_{2}\right)>0$, such that

$$
\left|\partial_{y} F(y)-\partial_{y} F(0)\right| \leq C_{5}|y|
$$

for all $y \in \mathbb{R}^{N}$ with $|y| \leq \delta_{2}$. Assuming $\|u\|_{s} \leq \delta_{2} / C_{1}$ entails

$$
\begin{aligned}
\left\|\partial_{x}^{\alpha}\left(F(u)-\partial_{u} F(0)\right)\right\| & \leq\left\|\left(\partial_{u} F(u)-\partial_{u} F(0)\right) \partial_{x}^{\alpha} u\right\|+\|R\| \\
& \leq\left\|\partial_{u} F(u)-\partial_{u} F(0)\right\|_{L^{\infty}}\|u\|_{s}+C_{3} C_{4}\left\|\partial_{x} u\right\|_{s-1} \\
& \leq \max \left\{C_{3} C_{4}, C_{5}\right\}\|u\|_{s}^{2} .
\end{aligned}
$$

Since $\alpha$ was arbitrary this estimate together with (A.1) leads the assertion for $\delta=\min \left\{\delta_{1}, \delta_{2}\right\} / C_{1}$.

A.2 Lemma. Let $n, N \in \mathbb{N}, s \geq s_{0}$ and $F \in C^{\infty}\left(\mathbb{R}^{N}, \mathbb{R}^{N \times N}\right)$. Then there exist $\delta, C=C(\delta)>0$ such that for all $u \in H^{s}\left(\mathbb{R}^{n}, \mathbb{R}^{N}\right)$ with $\|u\|_{s} \leq \delta$, $(F(u)-F(0)) u \in H^{s}$ and

$$
\|(F(u)-F(0)) u\|_{s} \leq C\|u\|_{s}^{2} .
$$

Proof. First note that there exist $\delta_{1}, C_{1}=C_{1}\left(\delta_{1}\right)>0$ such that

$$
|F(y)-F(0)| \leq C_{1}|y|
$$

for all $y \in \mathbb{R}^{N},|y| \leq \delta_{1}$ as well as $C_{2}>0$ such that

$$
\|v\|_{L^{\infty}} \leq C_{2}\|v\|_{s}
$$


for all $v \in H^{s}$. Now let $u \in H^{s},\|u\|_{s} \leq \delta_{1} / C_{2}$. Then

$$
\|F(u)-F(0)\| \leq C_{1}\|u\|_{s} .
$$

On the other hand by [6, Lemma 2.4] $\partial_{x} F(u) \in H^{s-1}$ and

$$
\left\|\partial_{x} F(u)\right\|_{s-1} \leq C_{3}\left\|\partial_{x} u\right\|_{s-1}
$$

for a $C_{3}=C_{3}\left(\delta_{1}\right)>0$. Hence $F(u)-F(0) \in H^{s}$ and

$$
\|F(u)-F(0)\|_{s} \leq C_{4}\|u\|_{s}
$$

for $\|u\|_{s} \leq \delta=\delta_{1} / C_{2}$. Now the assertion follows from [6, Lemma 2.4].

Conflict of interest: There is no conflict of interest.

\section{References}

[1] P. M. Dharmawardane, J. E. Muñoz Rivera, and S. Kawashima. Decay property for second order hyperbolic systems of viscoelastic materials. J. Math. Anal. and Appl., 366(2):621-635, 2010.

[2] H. Freistũhler. Godunov variables in relativistic fluid dynamics. arXiv:1706.06673.

[3] H. Freistühler and B. Temple. Causal dissipation and shock profiles in the relativistic fluid dynamics of pure radiation. Proc. $R$. Soc. A, 470:20140055, 2014.

[4] S. K. Godunov. An interesting class of quasilinear systems. Dokl. Akad. Nauk SSSR, 139:521-523, 1961.

[5] T. J. R. Hughes, T. Kato, and J. E. Marsden. Well-posed quasilinear second-order hyperbolic systems with applications to nonlinear elastodynamics and general relativity. Arch. Rational Mech. and Anal., 63(3):273-294, 1977.

[6] S. Kawashima. Systems of a Hyperbolic-Parabolic Composite Type, with Applications of Magnetohydrodynamics. PhD thesis, Kyoto University, 1983.

[7] S. Weinberg. Gravitation and Cosmology: Principles and Applications of the General Theory of Relativity. John Wiley \& Sons, New York, 1972. 